\title{
Welcome message from the Editor-in-Chief
}

\section{Ghazanfar Ali Safdar}

Senior Lecturer in Computer Networking, School of Computer Science and Technology, University of Bedfordshire, Park Square, Luton, LU1 3JU, United Kingdom

Received on 08 September 2017; published on 04 October 2017

Copyright (C) 2017 G.A. Safdar, licensed to EAI. This is an open access article distributed under the terms of the Creative Commons Attribution license (http://creativecommons.org/licenses/by/3.0/), which permits unlimited use, distribution and reproduction in any medium so long as the original work is properly cited.

doi:10.4108/eai.4-10-2017.153159

Welcome to September issue of the journal. Congratulations to the authors for their publications in the EAI Endorsed Transactions on Energy Web and Information Technologies.

EAI Endorsed Transactions on Energy Web and Information Technologies is a new open access scholarly archival journal that is committed to the timely publication of high-quality original research papers on all aspects of Energy efficient communication and Information Technologies. Original contributions that have not been published and are not currently under consideration by any other journal are solicited. All submissions will go through a rigorous peer-review process, and will be reviewed and evaluated by expert referees and the editorial board. Areas of interests include, but are not limited to:
- Green Data Centres and Data Storage

- Greener Smart Homes

- Green Smart Cities

- Greener Secure Web Transactions

- $\quad$ Energy Systems Modelling

- $\quad$ Energy Efficient Virtualized Systems

- Energy Efficient DSA (for CR Networks, D2D/M2M)

- Energy Efficient LAA (Licensed Assisted Access for $5 \mathrm{G}$ )

- $\quad$ Energy Aware Interference Mitigation in 4G Networks

- Energy Conservation and Mangement Strategies

- $\quad$ Telemetry and Power Engineering Based on Self-Organising Networks with Combined Information Transmission Channels

- Transportation and Electric Vehicles

- Green Communication Protocols

- $\quad$ Energy Efficient Routing Protocols

- Physical Layer Techniques for Energy Efficiency

- Energy Efficient Device-to-Device (D2D) Communications

- Energy-Aware Design for Small Cells and HetNets

- $\quad$ Energy Efficient Algorithms and Scheduling Techniques

- Green Massive MIMO Techniques

- Cross-Layer Energy Efficiency Optimisation

- Standardisation for Green Communications and Networking 
- Energy Efficient and Secure Implementation of Cryptographic Protocols

- $\quad$ Lightweight Cryptography

- Monitoring and Management of Energy Supply

- Online Learning Technologies in the Energy Sector

- Power Systems Regulations

- $\quad$ Power Systems Transmission Management

- $\quad$ Supply Chain Energy Management

This issue includes a collection of 5 outstanding papers (including editorial) that are contributed by leading researchers and cover a diversity of topics in the area of Energy Web and Information Technologies. We hope that you will find the papers timely, informative, and enjoyable.

\section{The papers are:}

\section{1: Energy Efficiency Led reduced CO2 Emission in Green LTE Networks}

\section{2: Extracting Academic Subjects Semantic Relations Using Collocations}

\section{3: Green Communications: Techniques and Challenges}

\section{4: Proposed a MAC Layer for Visible Light Communication}

The technological advancements in smart phones and their applications have rapidly raised the number of users and their data demands. To fulfill enlarged user's data requirements, Basestation (BS) engages their resources over prolong time intervals at the cost of increased power consumption. In parallel, operators are expanding network infrastructure by employing additional BSs which also adds in power consumption. This directly increases carbon emission ( $\mathrm{CO} 2)$ thus results in to more global warming. Therefore, Information and Communication Technology (ICT) has become major contributor in global warming while mobile communication is one of the key contributors within ICT. First paper investigates reduced $\mathrm{CO} 2$ emission through decreased power consumption in LTE networks. Proposed energy saving scheme is validated through the analysis of various performance related parameters in MATLAB. Results have proven that proposed scheme reduces $\mathrm{CO} 2$ emission by 2.10 tons per BS.

Second paper presents approach to analyze semantic content of academic subjects and its internal relations using statistically-based techniques for collocation extraction from large electronic educational text corpus. It offers a survey and analysis of some related corpusbased approaches to extract conceptual relations used for educational purpose and presents a technique for semantic search of collocations. The results of extended

Keyword search from British Academic Spoken English corpus using Sketch Engine searching software are presented. They are analysed with respect to types of generated keyword's collocations and semantic relations which they assign.

Third paper aligns very well to the scope of the journal and presents Greener communication techniques. Paper in this context gives an overview of important and well known greener communication methods and presents some interesting challenges too. Finally fourth paper presents a Medium access control layer for visible light communication. Paper presents hardware implementation and sound performance analysis. 
Ghazanfar A. Safdar received his B.Sc Hons. in Electrical Engineering from University of Engineering and Technology, Pakistan, and M.Eng in Computer Science and Telecommunications from ENSIMAG, INPG, France. He was awarded a PhD from Queen's University Belfast, UK, in 2005 for his work in the area of power-saving MAC protocols for the IEEE 802.11 family of wireless LANs. Moreover, he has worked as Research fellow at Queen's University Belfast on a project related to Wireless Networks security funded by EPSRC grant $\mathrm{EP} / \mathrm{C} 006976 / 1$. Currently, he is working as a Senior Lecturer in Computer Networking at the University of Bedfordshire, UK. His research interests mainly include cognitive radio networks, energy saving MAC protocols, security protocols for wireless networks, LTE networks, Interference mitigation, network modelling and performance analysis. He has also worked as R\&D Engineer with Carrier Telephone Industries (SIEMENS), Pakistan and Schlumberger France. Currently he is acting as EiC for EAI Transactions on Energy Web and Information technologies, he is also acting as Area Editor for Springer Wireless Networks. He has over 50 publications in top quality Journals and conferences in the area of wireless communication and networks. 